\title{
Identificação e Avaliação dos Espaços Livres de Uso Público da Região Administrativa 6 - Vitória - ES - Brasil'
}

\section{Identification and Evaluation of Space for Public Use in Administrative Region 6 - Vitória - ES - Brazil}

\section{Koffer, Amanda Lovatti Coelho'; Jesus, Luciana Aparecida Netto2; Conde, Karla Moreira33; Ramos, Larissa Andara4;}

\begin{abstract}
1 Universidade Federal do Espírito Santo (UFES), Vitória, Espírito Santo, Brasil, lovatti.amanda@gmail.com

2 Universidade Federal do Espírito Santo (UFES), luciana.a.jesus@ufes.br

3 Universidade Federal do Espírito Santo (UFES), karla.conde@ufes.br

4 Universidade Vila Velha (UVV), larissa.ramos@uvv.br
\end{abstract}

\begin{abstract}
RESUMO
A carência de espaços livres de uso público para o usufruto da população é resultado da evolução das cidades atuais. Em auxílio à verificação desses espaços na cidade de Vitória ES - Brasil, a presente pesquisa parte de uma rede colaborativa entre a Universidade Federal do Espírito Santo e Universidade Vila Velha, busca identificar, classificar e avaliar as praças pertencentes à região administrativa 6 - Goiabeiras. Após levantamento bibliográfico e mapeamento das praças, ocorreu um estudo para a adequação dos indicadores da ferramenta Índice de Caminhabilidade (iCam), os quais foram organizados em 4 categorias, respectivamente: Proteção e Segurança; Conforto e Imagem; Acessos e Conexões; e Sociabilidade, Usos e Atividades. Este artigo irá apresentar a avaliação correspondente à categoria "Conforto e Imagem", a qual verifica a situação do ambiente da praça e sua relação com as áreas verdes pertencentes a esta, que influenciam diretamente no conforto do usuário. Os resultados obtidos auxiliam no melhor entendimento da interface entre o usuário junto à cidade e sua relação com a natureza, bem como na elaboração de diretrizes e tomadas de decisões referentes à melhoria do espaço público.
\end{abstract}

Palavras-chave: Espaços livres de uso público, Avaliação de praças, Qualidade urbana.

\begin{abstract}
The lack of public spaces that are for population usufruct is a result of the evolution of the current cities. In support for the verification of these spaces in the city of Vitória - ES - Brazil, the present research of scientific initiation seeks to identify, classify and evaluate the squares belonging to the administrative region 6 - Goiabeiras. After a bibliographical survey and mapping of the squares, a study was carried out for the adequacy of the indexes of the

${ }^{1}$ KOFFER, Amanda Lovatti Coelho; JESUS, Luciana Aparecida Netto; CONDE, Karla Moreira; RAMOS, Larissa Andara. Identificação e Avaliação dos Espaços Livres de Uso Público da Região Administrativa 6 - Vitória - ES Brasil. In: II SIMPÓSIO NACIONAL DE GESTÃO E ENGENHARIA URBANA: SINGEURB, 2019, São Paulo. Anais... Porto Alegre: ANTAC, 2019.
\end{abstract}


Walkability Index, which were organized in 4 categories, respectively: Protection and Safety; Comfort and Image; Access and Connections; and Sociability, Uses and Activities. This article will present the evaluation corresponding to the category "Comfort and Image", which verifies the situation of the environment of the square and its relation with the green areas belonging to it, that directly influence the comfort of the user. The results obtained help to better understand the user's interface with the city and its relationship with nature, as well as in the elaboration of guidelines and decision-making regarding the improvement of the public space.

Keywords: Space for public use, Evaluation of squares, Urban quality.

\section{APRESENTAÇÃO}

As praças são atribuídas como elementos estruturantes da conformação urbana, a considerar o desenho da cidade. Robba \& Macedo (2010, p. 17) distinguem as praças dos demais espaços livres da cidade, limitando-as a "[...] espaços livres de edificações, públicos e urbanos, destinados ao lazer e ao convívio da população, acessíveis aos cidadãos e livres de veículos", a compactuar com a definição de Mora (2009 apud MACIEL, 2016), pela qual as praças se estabelecem como elementos básicos da estrutura da cidade, assim como são as ruas, responsáveis pela representação da cultura e história dos seus respectivos usuários. Por conseguinte, Brandão Alves (2003), conceitua as praças a partir da capacidade de acolher atividades sociais, sendo pontos de referência para os que se deslocam pela cidade, são marcos e pontos nodais (LYNCH, 1960) importantes para o desenho da paisagem $e$ orientação urbana.

Além das funções sociais, os espaços livres, ainda, podem possuir funções estéticas e ecológicas (HANNES, 2016). As funções estéticas se encarregam da construção da paisagem, refletindo a imagem da cidade, embelezando-a e diversificando-a. As funções ecológicas são diretamente ligadas às áreas verdes pelos quais podem ou não serem dotados. A presença de vegetação e solo permeável garantem benefícios não somente para a qualidade de vida da região, como também promove benefícios à cidade, em questões como a drenagem do solo urbano, por exemplo.

O aprimoramento dos espaços verdes urbanos tem potencial para amansar os efeitos da urbanização de forma sustentável, tornando as cidades mais atraentes e acima de tudo mais saudáveis para a população (MENDES, 2017). Há, ainda, a compreensão dos benefícios ambiental, social e economicamente. No que tange os benefícios ambientais, de acordo com Mendes (2017), esses podem ser evidenciados na regulação do clima, redução dos efeitos de "ilhas de calor", diminuição de energia pela redução do uso de equipamentos de conforto térmico, redução de níveis de polvição do ar, aumento da umidade relativa do ar, conservação da biodiversidade e manutenção do ciclo hidrológico, à medida que se garante a permeabilidade do solo.

Considerando a potencial formação das praças a partir do próprio desenho da cidade e apesar dos diversos benefícios provenientes dessas para a vida urbana, percebe-se a necessidade da averiguação da eficiência destes espaços, de acordo com a experiência do usuário, tanto em quantidade como em qualidade. Como auxílio ao melhor entendimento do funcionamento das praças, discute-se neste artigo a seleção e avaliação de 11 praças, a partir da adequação da ferramenta Índice de Caminhabilidade (iCam), desenvolvida pelo Instituto de Pesquisas de Transporte e Desenvolvimento (ITDP) (ITDP Brasil, 2018).

A discussão se baseia na apropriação do espaço público, aliado às demandas sustentáveis e é realizada conforme parâmetros apresentados pelo projeto de pesquisa "Análise socioambiental de espaços livres de uso público na Grande Vitória", promovido por um grupo de pesquisa organizado através da parceria entre duas universidades do Espírito Santo - Brasil e que busca, a partir da avaliação de praças, discutir as diferenças de tratamento do espaço em diferentes pontos da região metropolitana de Vitória - ES. 
Os espaços analisados estão localizados em 7 bairros, com os quais se caracteriza a região administrativa 6 - Goiabeiras, conforme a lei nº.611 de 2014, sendo eles: Aeroporto, Antônio Honório, Goiabeiras, Jabour, Maria Ortiz, Segurança do Lar e Sólon Borges. A região é marcada por referências características tanto para localização como para composição da paisagem, como o Aeroporto Eurico Salles e o campus da Universidade Federal do Espírito Santo, e conta com aproximadamente $5,7 \%$ da população do município.

\section{MÉTODO}

A produção do trabalho passou por um levantamento bibliográfico prévio, com o qual se possibilitou o conhecimento dos trabalhos desenvolvidos na área, suas metodologias e abordagens. O levantamento se tornou fundamento para a conceituação do trabalho e contextualização da área a ser analisada no estudo de caso, realizados com o auxílio do Plano Diretor Urbano do município e dados fornecidos pelo Instituto Brasileiro de Geografia e Estatística (IBGE).

A contextualização propiciou o mapeamento dos espaços a serem analisados, realizado com o auxílio de ferramentas de geoprocessamento e Sistema de Informação Geográfica (SIG), no software ArcGIS, versão 10.4.1, a partir de dados cedidos pela prefeitura.

A base utilizada para a avaliação das praças foi a ferramenta Índice de Caminhabilidade (iCam), organizada pelo ITDP Brasil (2018), ferramenta essa destinada à avaliação de vias, com a qual, estudos e discussões em grupo possibilitaram a adequação e melhor organização para a aplicação no espaço das praças, objeto de estudo da pesquisa.

O método avaliativo é organizado em categorias, atributos e indicadores. Conforme define - iCam (2018), as categorias apresentam os principais parâmetros de referência para a avaliação. São essas que distribuem, em um primeiro momento, as pontuações adotadas. Os atributos foram criados como "sub-categorias", responsáveis pela maior organização e especificidade do indicador pertencente a esta. Já os indicadores serão os responsáveis pela qualificação unitária do desempenho apresentado pelo objeto de estudo.

Os 36 indicadores estudados para esta pesquisa estão organizados em 11 atributos, dispostos em 4 respectivas categorias, sendo elas "Proteção e Segurança", "Conforto e Imagem", "Acessos e Conexões" e "Sociabilidade, Usos e Atividades", estabelecidas conforme o Guia dos Espaços Públicos (2015), referência utilizada para melhor agrupar e organizar os atributos e indicadores. Este artigo tem como objetivo a apresentação dos resultados obtidos na categoria "Conforto e Imagem", que visa entender o ambiente da praça e sua relação com a natureza, os quais influem diretamente no conforto do usuário. O Quadro 1 apresenta os indicadores pertencentes à categoria a ser avaliada, sendo identificados em cinza os indicadores adicionados além da matriz original do iCam. A inclusão de novos atributos e indicadores deve-se a necessidade de adequação do método avaliativo, considerando que as praças possuem locais de permanência, diferente das calçadas, cuja função principal é permitir o adequado fluxo dos pedestres.

Para a medição dos indicadores, foram utilizados os parâmetros apresentados pelo iCam. Assim, alguns indicadores puderam ser medidos por observação, como "Coleta de Lixo" e "Assentos", em outros indicadores foram utilizados equipamentos específicos, como "Polvição Sonora", na utilização de decibelímetro, admitindo-se a utilização de aplicativos previamente calibrados e testados. As avaliações correspondentes à sombra consideraram o cálculo da área de projeção de vegetação e abrigos em relação a área total da praça, possibilitados a partir do uso de imagens de satélite do programa Google Earth.

Para a classificação, adotou-se o sistema de pontuação do próprio iCam, no qual, a partir dos respectivos parâmetros, dá-se nota de 0 (zero) a 3 (três) para o atributo a ser estudado, conforme Quadro 2. 
Quadro 1 - Atributos e Indicadores pertencentes à categoria Conforto e Imagem

\begin{tabular}{|c|c|}
\hline \multicolumn{2}{|c|}{ Categoria: Conforto e Imagem } \\
\hline Atributos & Indicadores \\
\hline \multirow{4}{*}{ Ambiente } & Coleta de Lixo \\
\hline & Polvição Sonora \\
\hline & Sombra e Abrigo \\
\hline & Sombra e Abrigo em Área Específica \\
\hline \multirow{3}{*}{$\begin{array}{c}\text { Áreas Verdes/ Cobertura } \\
\text { Vegetal }\end{array}$} & Área de Sombra de Copa de Árvore \\
\hline & $\begin{array}{l}\text { Área de Sombra de Copa de Árvore em Área } \\
\qquad \text { Específica }\end{array}$ \\
\hline & Cobertura Vegetal \\
\hline Espaços para sentar & Assentos \\
\hline
\end{tabular}

Fonte: As autoras, 2019

Quadro 2 - Pontuação para Avaliação à partir de Indicadores

\begin{tabular}{|c|c|c|c|}
\hline Pontuação 3 & $\begin{array}{c}\text { Pontuação } 2 \text { até } \\
\mathbf{2 , 9}\end{array}$ & $\begin{array}{c}\text { Pontuação } 1 \text { até } \\
\mathbf{1 , 9}\end{array}$ & $\begin{array}{c}\text { Pontuação } 0 \text { até } \\
\mathbf{0 , 9}\end{array}$ \\
\hline Ótimo & Bom & Suficiente & Insuficiente \\
\hline
\end{tabular}

Fonte: adaptado de Índice de Caminhabilidade, 2018

Em paralelo ao estudo dos indicadores, foram selecionados os espaços a serem trabalhados. Para tal, caracterizou-se para este estudo, as praças, espaços livres de uso público com área total maior ou igual a 450m² (BUCCHERI FILHO; NUCCl, 2006). As aplicações foram testadas em visitas de campo e análise de dados, os quais foram organizados para que seja assegurada veracidade em posterior comparação de resultados.

\section{IDENTIFICAÇÃO DOS ESPAÇOS LIVRES DE USO PÚBLICO}

A partir do mapeamento, na região administrativa 6 - Goiabeiras foram identificados 20 espaços livres de uso público. São 11 praças públicas, 8 espaços livres com área menor que 450m² (BUCCHERI FILHO; NUCCl, 2006) e 3 espaços potenciais distribuídos pela região, como listado no Quadro 3. A prefeitura classifica os espaços livres apresentados como praças, porém, ao definir o critério da área mínima, desconsideram-se esses para posterior análise. 
Quadro 3 - Lista de Espaços Livres Identificados na Região Administrativa 6

\begin{tabular}{|c|c|}
\hline Bairro & Espaços Livres de Uso Público \\
\hline Aeroporto & - \\
\hline \multirow{3}{*}{ Goiabeiras } & Praça Darcy José de Sá Filho \\
\hline & Campo do Hi-fi \\
\hline & Praça da Familia \\
\hline Antônio Honório & Praça Coronel Francisco P. do Nascimento \\
\hline Segurança do Lar & - \\
\hline \multirow{3}{*}{ Sólon Borges } & Praça Adriano Mathielo \\
\hline & Praça Odilon Grijo \\
\hline & Praça Professor Colares Júnior \\
\hline Maria Ortiz & Praça Salvador da Silva Campos \\
\hline \multirow{3}{*}{ Jabour } & Praça Jenuíno Geraldo dos Santos \\
\hline & Praça Leonardo Pereira da Silva \\
\hline & Praça Palestina Livre \\
\hline
\end{tabular}

Fonte: As autoras, 2019

Os bairros Aeroporto e Segurança do Lar não possuem espaços livres identificados, porém, atribuindo-se um raio de abrangência de $400 \mathrm{~m}$ aos espaços existentes na região administrativa, percebe-se que esses atendem toda a população residente, pelo fato de algumas praças abrangerem além do interior de seus próprios bairros, também os vizinhos.

\section{APLICAÇÃO DOS INDICADORES AMBIENTAIS}

Em visitas de campo e análises a partir dos dados coletados, foram obtidas as pontuações apresentadas no Quadro 4 para a categoria Conforto e Imagem.

Observando os resultados, percebe-se a irregularidade das praças de uma mesma regional e até mesmo dentro de um único bairro. Aproximadamente $27 \%$ das praças apresentam resultado "bom", 63\% das praças se apresentam como "suficientes" e 9\% apresentam pontuação "insuficiente". O bairro Goiabeiras, por exemplo, apresenta as 3 praças com pontuações diferentes, e, ainda a praça "Campo do Hi-fi" chama atenção por sua pontuação beirando o limite inferior, com quase todos os indicadores zerados. As demais praças apresentam suficiência, porém é necessária atenção a elas, visto que ser suficiente não atribui valor significativo para o conforto do transeunte.

O panorama geral pode dizer respeito à preservação da vegetação original proveniente da região, a qual se situa próxima a grande área de preservação do município. Essa vegetação, aliada ao bom cuidado com a praça e seu entorno, junto à atribuição de mobiliário, conferem um ambiente agradável, propício ao convívio dos que ali possam se encontrar. As praças que não apresentam essas características se tornam não somente desagradáveis, como repelem o usuário, por não possuir condições favoráveis à sua permanência. 
Quadro 4 - Resultado da avaliação das praças para a categoria Conforto e Imagem

\begin{tabular}{|c|c|c|c|c|c|c|c|c|c|c|c|c|c|c|c|c|c|c|c|c|c|c|}
\hline \multirow{3}{*}{$\begin{array}{l}\text { Indicadores } \\
\text { Coleta de Lixo }\end{array}$} & \multicolumn{6}{|c|}{ Bairro Goiabeiras } & \multirow{2}{*}{\multicolumn{2}{|c|}{$\begin{array}{l}\text { Bairro } \\
\text { Antônio } \\
\text { Honório }\end{array}$}} & \multicolumn{6}{|c|}{ Bairro Sólon Borges } & \multirow{2}{*}{\multicolumn{2}{|c|}{$\begin{array}{l}\text { Bairro } \\
\text { Maria } \\
\text { Ortiz }\end{array}$}} & \multicolumn{6}{|c|}{ Bairro Jabour } \\
\hline & \multirow[t]{2}{*}{ 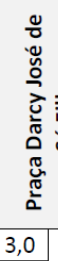 } & & \multicolumn{2}{|c|}{$\begin{array}{l}\frac{1}{1} \\
\frac{1}{1} \\
0 \\
0 \\
0 \\
0 \\
\frac{0}{E} \\
0 \\
0\end{array}$} & \multicolumn{2}{|c|}{ 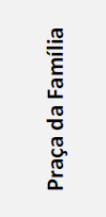 } & & & \multicolumn{2}{|c|}{ 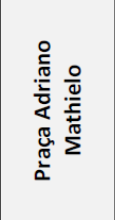 } & \multicolumn{2}{|c|}{ 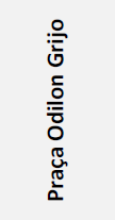 } & \multicolumn{2}{|c|}{ 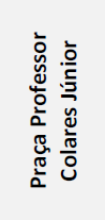 } & & & \multicolumn{2}{|c|}{ 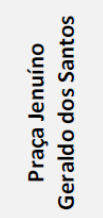 } & \multicolumn{2}{|c|}{ 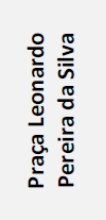 } & \multicolumn{2}{|c|}{ 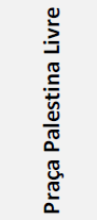 } \\
\hline & & \multirow{4}{*}{2,3} & 3,0 & \multirow{4}{*}{1,5} & 3,0 & \multirow{4}{*}{3,0} & 3,0 & \multirow{4}{*}{2,0} & 2,0 & \multirow{4}{*}{1,8} & 2,0 & \multirow{4}{*}{2,8} & 3,0 & \multirow{4}{*}{2,0} & 2,0 & \multirow{4}{*}{2,8} & 0,0 & \multirow{4}{*}{1,0} & 3,0 & \multirow{4}{*}{1,8} & 3,0 & \multirow{4}{*}{2,0} \\
\hline Poluição Sonora & 2,0 & & 3,0 & & 3,0 & & 1,0 & & 3,0 & & 3,0 & & 3,0 & & 3,0 & & 3,0 & & 3,0 & & 3,0 & \\
\hline Sombra e Abrigo & 1,0 & & 0,0 & & 3,0 & & 1,0 & & 2,0 & & 3,0 & & 2,0 & & 3,0 & & 1,0 & & 1,0 & & 2,0 & \\
\hline $\begin{array}{l}\text { Sombra e Abrigo } \\
\text { em Área Específica }\end{array}$ & 3,0 & & 0,0 & & 3,0 & & 3,0 & & 0,0 & & 3,0 & & 0,0 & & 3,0 & & 0,0 & & 0,0 & & 0,0 & \\
\hline $\begin{array}{c}\text { Área de Sombra } \\
\text { de Copa de Árvore }\end{array}$ & 0,0 & \multirow{3}{*}{1,7} & 0,0 & \multirow{3}{*}{0,0} & 3,0 & \multirow{3}{*}{3,0} & 1,0 & \multirow{3}{*}{2,0} & 2,0 & \multirow{3}{*}{1,7} & 3,0 & & 2,0 & & 0,0 & & 1,0 & & 1,0 & & 2,0 & \\
\hline $\begin{array}{l}\text { Área de Sombra } \\
\text { de Copa de Árvore } \\
\text { em Área Específica }\end{array}$ & 2,0 & & 0,0 & & 3,0 & & 3,0 & & 0,0 & & 3,0 & 3,0 & 0,0 & 1,7 & 3,0 & 1,7 & 0,0 & 1,3 & 0,0 & 1,3 & 0,0 & 1,7 \\
\hline Cobertura Vegetal & 3,0 & & 0,0 & & 3,0 & & 2,0 & & 3,0 & & 3,0 & & 3,0 & & 2,0 & & 3,0 & & 3,0 & & 3,0 & \\
\hline Assentos & 1,0 & 1,0 & 0,0 & 0,0 & 2,0 & 2,0 & 1,0 & 1,0 & 2,0 & 2,0 & 1,0 & 1,0 & 1,0 & 1,0 & 2,0 & 2,0 & 0,0 & 0,0 & 2,0 & 2,0 & 1,0 & 1,0 \\
\hline Média das Praças & 1 & 9 & & 8 & & 9 & 1, & 9 & 1 & & & & 1 & & 2 & & 1, & & & 6 & & 8 \\
\hline Média dos Bairros & & & & 5 & & & 1, & 9 & & & & & & & 2 & & & & 1, & & & \\
\hline
\end{tabular}

Fonte: As autoras, 2019

Pensar no conforto do usuário é pensar em sua apropriação do espaço, e a influência da natureza nesse pensamento é de extrema importância para se qualificar o ambiente. Por isso, os resultados obtidos na região administrativa estudada sugerem a necessidade de maior atenção ao que se refere relação do ambiente das praças com a boa atmosfera a qual consegue-se com o cuidado com a camada ecológica da cidade.

\section{CONSIDERAÇÕES FINAIS}

Verifica-se que o campo de avaliação de espaços livres carece de ferramentas hábeis e de aplicação direta. A metodologia apresentada, além de reunir parâmetros do índice de Caminhabilidade (iCam), propôs novos indicadores a fim de especificar ainda mais as verificações, com as quais podem ser tomadas diretrizes pontuais para a resolução dos potenciais problemas serem encontrados nas praças.

Assim, considerando a aplicação da ferramenta na região administrativa 6 - Goiabeiras, no que tange a categoria Conforto e Ambiente, se torna possível a discussão de pontos importantes, alvo de altos e baixos na avaliação das praças, como é o caso da necessidade do projeto prévio dos espaços livres, preservação da vegetação original e manutenção da praça, a qual atribui características importantes para o conforto do usuário, a se considerar, por exemplo, sombreamento, limpeza, visuais e sons agradáveis.

\section{AGRADECIMENTOS}

As autoras agradecem à Universidade Federal do Espírito Santo (UFES) pelo apoio concedido à primeira autora deste artigo.

\section{REFERÊNCIAS}

BRANDÃO ALVES, F. Avaliação da qualidade do espaço público urbano. Proposta

Metodológica. Fundação Calouste Gulbenkian. Fundação para a Ciência e Tecnologia. Lisboa, 2003.

BRASIL, ITDP. Índice de Caminhabilidade Ferramenta, Versão 2.0. Rio de Janeiro, 2018. 
BUCCHERI FILHO, A.T.; NUCCl, J.C. Open spaces, green areas and tree canopy coverage in the Alto da XV district, Curitiba/PR. Revista do Departamento de Geografia, n. 18, 2006. p. 4859.

CAVALHEIRO, F. et al. Proposição de Terminologia para o Verde Urbano. Boletim Informativo da Sociedade Brasileira de arborização urbana. SBAU: Ano VII, Rio de Janeiro, 1999.

HANNES, Evy. Espaços abertos/espaços livres: um estudo de tipologias. Paisagem e Ambiente, n. 37, p. 121-144, 2016.

HEEMANN, Jenifer; SANTIAGO, P. Caiuby. Guia do espaço público para inspirar e transformar. Mountain View (CA), USA, 2015.

IBGE. Instituto Brasileiro de Geografia e Estatística. CENSO DEMOGRÁFICO 2010.

Características da população e dos domicílios: resultados do universo. Rio de Janeiro: IBGE, 2010.

LYNCH, Kevin. A Imagem da Cidade, 3a edição. WMF Martins Fontes. São Paulo, 2011.

MACIEL, Mariana Altoé. Uma proposta de lista de verificação para a avaliação de praças.

2016. Dissertação de Mestrado. Universidade Federal do Espírito Santo. Disponível em:

<http://repositorio.ufes.br/handle/10/10047>. Acesso em: 06, Set, 2018.

MENDES, Alexandra Filipa Letra. Avaliação da disponibilidade e acessibilidade a espaços

verdes em quatro áreas urbanas: Lisboa, Porto, Braga e Coimbra. 2017. Dissertação de

Mestrado. Universidade do Porto. Disponível em: https://repositorio-

aberto.up.pt/bitstream/10216/108724/2/229301.pdf>. Acesso em: 31, Ago, 2018.

ROBBA, Fábio; MACEDO, Silvio S. Praças brasileiras: public squares in Brazil. 3. ed. São Paulo: Edusp, 2010. 